Short communication

\title{
A novel de novo deletion of chromosome 7 [46,XX,del(7)(p14.2 p15.1)] in a child with feeding problems
}

\author{
Henedina Antunes ${ }^{\text {a,b,f,* }}$, Jean-Pierre Gonçalves ${ }^{\mathrm{a}, \mathrm{c}}$, Esmeralda Silva ${ }^{\mathrm{d}}$, Natália Teles ${ }^{\mathrm{e}}$ \\ a Pediatric Gastroenterology Unit, Braga Hospital, Portugal \\ ${ }^{\mathrm{b}}$ Life and Health Sciences Research Institute (ICVS), School of Health Sciences, University of Minho, Braga, Portugal \\ c Community Health, School of Health Sciences, University of Minho, Portugal \\ ${ }^{\mathrm{d}}$ Neonatology, Braga Hospital, Braga, Portugal \\ e Cytogenetic Unit, Medical Genetic Institute, Porto, Portugal \\ ${ }^{\mathrm{f}}$ ICVS/3B's, PT Government Associate Laboratory, Braga/Guimarães, Portugal
}

\section{A R T I C L E I N F O}

\section{Article history:}

Accepted 18 April 2012

Available online 29 April 2012

\section{Keywords:}

Phenotype

Interstitial

Karyotype

\begin{abstract}
A B S T R A C T
The phenotype and severity of symptoms associated with deletions on chromosome 7 are directly proportional to the size of the deleted segment. Distal and interstitial deletions have been described in 40 cases. In this report the authors aim to report a child with a novel de novo interstitial deletion on chromosome 7 , with the following karyotype: 46,XX,del(7)(p14.2 p15.1).

We described a female, born at 38 weeks with intrauterine growth restriction and feeding problems with episodes of cyanosis after feedings and failure to thrive. Physical examination showed low implantation of ears, hypertelorism, oblique palpebral fissures, retrognathia, and palate ogived, with insertion anomalies of the toes, poor facial expression and mild axial hypotonia. Transfontanelar ultrasound, magnetic resonance imaging, bronchofibroscopy and metabolic studies were normal. She was hospitalized until the 32nd day of life. She started speech therapy and presented improvements in swallowing. The percutaneous endoscopic gastrostomy was removed at 36 months. She had recurrent urinary tract infection with normal dimercaptosuccinic acid but with a vesicoureteral reflux (grade III). Imagiological studies revealed a bilateral osteonecrosis of femoral epiphysis (Legg-Calvé-Perthes disease).

Currently (6 years-old), she is being normally fed (body mass index $=15.8 \mathrm{~kg} / \mathrm{m}^{2}$ ). Her weight is $16.4 \mathrm{~kg}$ ( $3 \mathrm{rd}$ centile) and length is $105 \mathrm{~cm}$ (3rd to 5 th centiles). She has a mild delay of psychomotor development impairment and some speech problems.

This is the first case report of a patient with this de novo small interstitial deletion on chromosome 7 . This rare chromosomal abnormality was associated with severe feeding problems in the first years of life.
\end{abstract}

(c) 2012 Elsevier B.V. All rights reserved.

\section{Introduction}

Deletions on chromosome 7 have been associated with a range of phenotypes including craniofacial malformations (frontal prominent, craniosynostosis, microcephaly, malformed ears, changes in the eyes and eyelids), congenital heart problems, distal limbs and genitalia malformations and moderate to severe retardation of psychomotor development (Chotai et al., 1994; Hinkel et al., 1988; Schwarzbraun et al., 2006). The phenotype and severity of symptoms associated with deletions on chromosome 7 are directly proportional to the

Abbreviations: IUGR, intrauterine growth retardation; PEG, percutaneous endoscopic gastrostomy; aCGH, array comparative genomic hybridization; UTI, urinary tract infection; VUR, vesicoureteral reflux; 99mTc-DMSA, technetium-99m-labeled dimercaptosuccinic acid.

* Corresponding author at: Braga Hospital, Sete Fontes, S.Victor, 4710-243 Braga, Portugal. Tel.: + 351 253027000; fax: + 351253027999 (hospital).

E-mail address: henedinaantunes@gmail.com (H. Antunes). size of the deleted segment (Kosaki et al., 2005). Distal and interstitial deletions are described in 40 cases (Marks et al., 1985; Moedjono et al., 1978).

We experienced a case of feeding problems in a child with a novel de novo deletion on chromosome 7: 46,XX,del(7)(p14.2 p15.1).

\section{Patient and methods}

\subsection{Patient}

The patient is a female newborn referred for evaluation of dysmorphic features and intrauterine growth retardation (IUGR).

She was the second child of healthy and non-consanguineous parents. The family history is negative for birth defects, mental retardation, infant deaths or metabolic disorders.

Pregnancy, labor and delivery were uncomplicated. She was born at term. Her birth weight was $2480 \mathrm{~g}$ (3rd to 10th centiles), her 


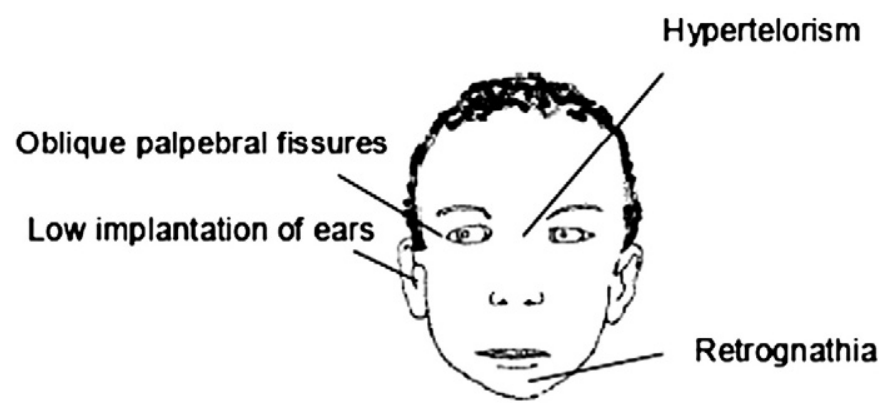

Fig. 1. Patient phenotype diagram showing a low implantation of ears, hypertelorism, oblique palpebral fissures and retrognathia.

length was $45.5 \mathrm{~cm}$ (3rd to 10th centiles) and her head circumference measured $34 \mathrm{~cm}$ (50th centile).

On newborn examination, notable dysmorphic facial features include a low implantation of ears, hypertelorism, oblique palpebral fissures, retrognathia, palate ogived, toe insertion anomalies, poor facial expression and mild axial hypotonia (Fig. 1).

Feeding difficulty and failure to thrive in her neonatal period were reported and a percutaneous endoscopic gastrostomy (PEG) was performed.

A transfontanelar ultrasound, magnetic resonance imaging, chest radiography, bronchofibroscopy and metabolic studies were normal.

\subsection{Cytogenetic analysis}

Classical karyotyping using conventional high resolution GTG banding (Hou and Wang, 1996) of the proband's peripheral blood revealed a small de novo interstitial deletion on the short arm of chromosome 7. Array comparative genomic hybridization (aCGH) techniques were performed as previously described (Buffart et al., 2008), in order to complement the cytogenetic findings, and define accurately the deletion breakpoints and the extent of the deletion. Genomic DNA was prepared from peripheral blood lymphocytes by a simple salting-out procedure. The analysis was done using the Nexus Copy Number 6.0 software with FASST2 Segmentation algorithm. The array design, database consultation and comparative analysis were performed using genome build 36.1/HG18.

Results showed a deletion in 7p15.3 $\rightarrow$ p15.1 (Figs. 3 and 4). Since the phenotypically normal parents showed normal karyotype, this abnormality was considered de novo.

Thus, the final karyotype could be defined as 46,XX,del(7)(p15.3p15.1).arr 7p15.3p15.1(20,469,062-30,582,316)×1 dn.

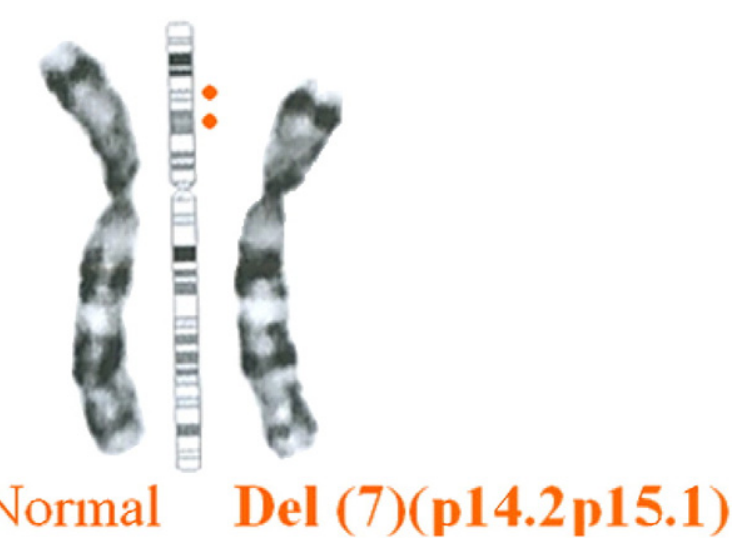

Fig. 3. Partial karyotype of the proband, showing del(7)(p14.1p15.1) after CTG. : Deleted region.

\section{Results}

The patient was hospitalized until the 32nd day of life, and presented with lack of coordination in sucking and swallowing.

Developmental delay including speech delay was noted in early infancy. She started speech therapy and presented improvements in swallowing. Percutaneous endoscopic gastrostomy tube was removed at 36 months. She had recurrent urinary tract infection (UTI) and vesicoureteral reflux (VUR) grade III. She had undergone further investigation by technetium-99m-labeled dimercaptosuccinic acid (99mTc-DMSA) scintigraphy that was normal. She has taken UTI antibiotic prophylaxis until the clinical investigation was complete.

Imagiological studies revealed a bilateral osteonecrosis of femoral epiphysis (Legg-Calvé-Perthes disease) (Fig. 2). She had no growth hormone or thyroid deficiency. The abdominal and neurological examinations were normal.

She underwent catheterism for treatment of patent ductus arteriosus.

At 4 years-old she showed a mild developmental delay (general quotient (GQ): 74.1) and some speech problems. She had some difficulties in fine movements and language expression, in comparison with normal children of the same age. At 6 years-old she was submitted to a bilateral epiphysiodesis of the greater trochanter to stabilize the relationship between the greater trochanter and the femoral head and neck regions.

Presently (6 years-old), she feeds normally (body mass index $=$ $15.8 \mathrm{~kg} / \mathrm{m}^{2}$ ). Her weight is $16.4 \mathrm{~kg}$ (3rd centile) and her length is $105 \mathrm{~cm}$ (3rd to 5 th centiles). She has a mild delay of psychomotor development impairment and some speech problems.

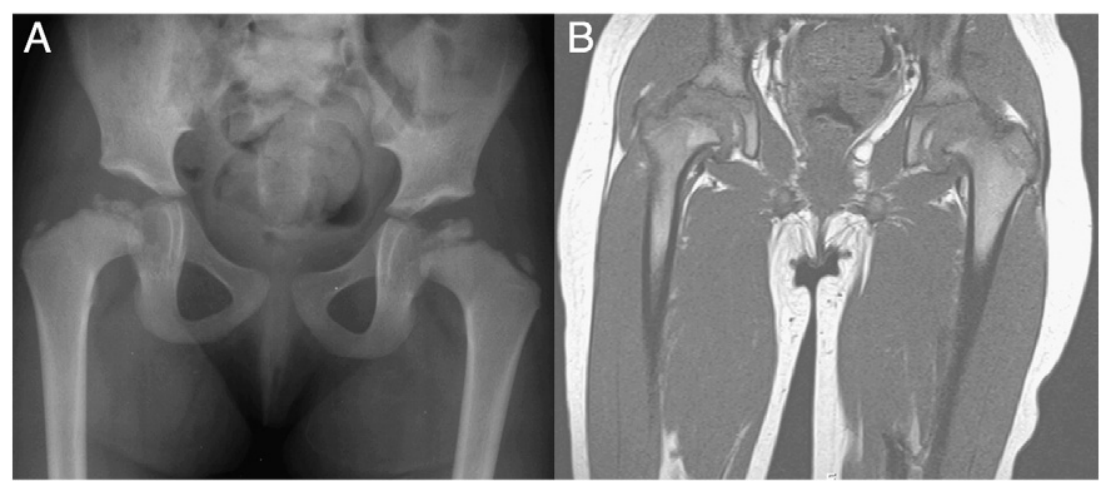

Fig. 2. Irregularity of the cortex of the bilateral femoral heads, consistent with the Legg-Calvé-Perthes disease at age of 4 years-old (pelvis X-ray (A) and magnetic resonance imaging (B)). 


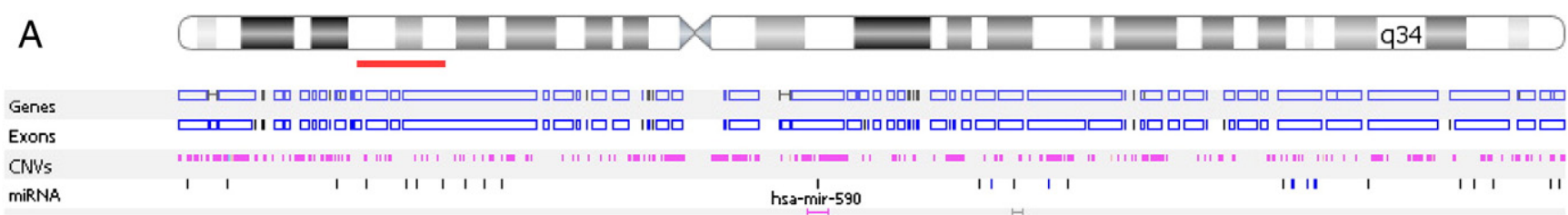

$\mathrm{B}$

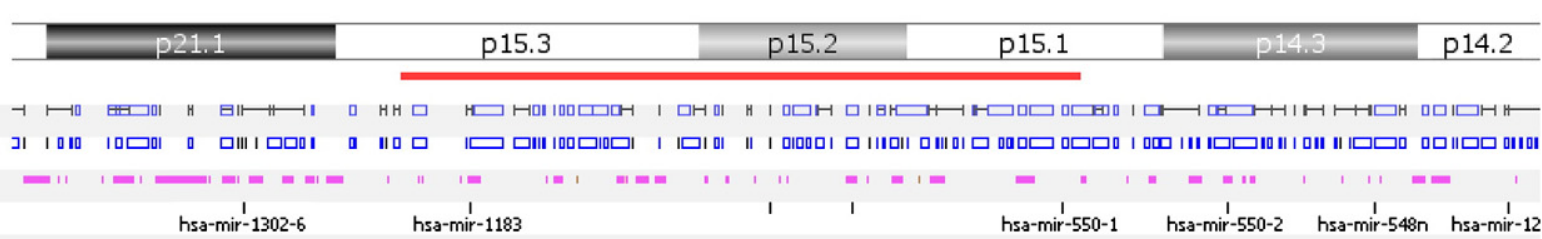

Fig. 4. Microarray analysis showing a loss of the short arm of chromosome 7 at bands p15.3p15.1, with a hemizygous loss of 101,132 and 254 nucleotides. A) Overview, B) zoomed view.

\section{Discussion}

When evaluating a newborn with peculiar phenotype and IUGR it is mandatory to perform chromosomal studies. In our patient, the genetic study revealed a novel de novo small interstitial deletion on chromosome 7, thus establishing the following karyotype: 46,XX $\operatorname{del}(7)(\mathrm{p} 14.2 \mathrm{p} 15.1)$.

This is probably the first case report of a patient with this deletion. The phenotypic spectrum described in this case report is consistent with other related cases of distal and interstitial deletions on chromosome 7. Our patient showed craniofacial malformations, VUR, mild axial hypotonia, developmental delay, feeding difficulty and failure to thrive.

She had no trauma or fever and so the Legg-Calvé-Perthes disease observed in this patient could probably be associated with this deletion. Nonetheless this association has not been reported, other studies have showed an association between deletion on chromosome 7 and skeletal disorders.

This rare abnormality was associated with severe feeding problems in the first years of life. In this case a PEG was a transient and efficient solution. This condition seems to be associated with a mild developmental delay and speech problems. As described in other studies the phenotype and severity of symptoms associated with deletions on chromosome 7 appear to be directly proportional to the number of deleted chromosomal regions (Chotai et al., 1994).

There is a significant association between $7 p$ deletions and craniosynostosis that was not observed in our case (Aughton et al., 1991; Chotai et al., 1994; Reardon et al., 1993). The precise location and extension of the deletion required for craniosynostosis is still unknown as some cytogeneticists indicate $7 \mathrm{p} 15$ as the likely critical area while others consider the 7p21 region to be more important (Aughton et al., 1991; Brueton et al., 1992).

Knowledge about clinical anomalies associated with deletions of chromosome 7 are still scarce and so we hope this case report could represent an improvement to better characterize this genetic disorder.

\section{Acknowledgments}

We would like to thank the patient's family for consenting to this publication and for their kind cooperation during the clinical investigations of their child. The authors also thank Professor Patrícia Maciel, Fátima Lopes and Dr. L. Van Maldergem for their help during this case study.

\section{References}

Aughton, D.J., Cassidy, S.B., Whiteman, D.A., Delach, J.A., Guttmacher, A.E., 1991. Chromosome $7 \mathrm{p}$-syndrome: craniosynostosis with preservation of region $7 \mathrm{p} 2$. Am. J. Med. Genet. 40, 440-443.

Brueton, L.A., van Herwerden, L., Chotai, K.A., Winter, R.M., 1992. The mapping of a gene for craniosynostosis: evidence for linkage of the Saethre-Chotzen syndrome to distal chromosome 7p. J. Med. Genet. 29, 681-685.

Buffart, T.E., et al., 2008. Across array comparative genomic hybridization: a strategy to reduce reference channel hybridizations. Genes Chromosomes Cancer 47, 994-1004.

Chotai, K.A., et al., 1994. Six cases of 7p deletion: clinical, cytogenetic, and molecular studies. Am. J. Med. Genet. 51, 270-276.

Hinkel, G.K., Tolkendorf, E., Bergan, J., 1988. 7p-deletion syndrome. Monatsschr. Kinderheilkd. 136, 824-827.

Hou, J.W., Wang, T.R., 1996. Chromosomal rearrangements detected by FISH and Gbanding. J. Formos. Med. Assoc. 95, 686-691.

Kosaki, R., Higuchi, M., Mitsui, N., Matsushima, K., Ohashi, H., Kosaki, K., 2005. Deletion involving the TWIST locus and the HOXA cluster: a contiguous gene syndrome on 7p? Congenit Anom (Kyoto) 45, 35-38.

Marks, K., Hill, L., Chitham, R.G., Whitehouse, W.L., 1985. Interstitial deletion of chromosome 7p detected antenatally. J. Med. Genet. 22, 316-318.

Moedjono, S.J., Funderburk, S.J., Sparkes, R.S., 1978. Chromosome 7 short-arm interstitial deletion ( $\mathrm{p} 14)$. Hum. Genet. 44, 51-57.

Reardon, W., McManus, S.P., Summers, D., Winter, R.M., 1993. Cytogenetic evidence that the Saethre-Chotzen gene maps to 7p21.2. Am. J. Med. Genet. 47, 633-636.

Schwarzbraun, T., et al., 2006. Genomic analysis of five chromosome 7p deletion patients with Greig cephalopolysyndactyly syndrome (GCPS). Eur. J. Med. Genet. 49, 338-345. 